\title{
La estructura comunicativa y social de los cuerpos académicos en México
}

\author{
The communicative and social structure \\ of the academic bodies in Mexico
}

\author{
Augusto David Beltrán Poot \\ Universidad Autónoma de Yucatán, México \\ dbeltran@correo.uady.mx \\ Código Orcid: https://orcid.org/0000-0002-6081-8096
}

\begin{abstract}
Resumen
El trabajo tiene como objetivo describir las estructuras comunicativas y sociales que se encuentran en los grupos de investigadores que configuran los cuerpos académicos en México. Se recurre a un estudio cualitativo-fenomenológico, donde se realizaron entrevistas y observaciones en un contexto particular, así como el análisis de 150 productos académicos sobre el tema. Los resultados describen que los cuerpos académicos mantienen una estructura social y comunicativa cerrada hacia fuera, tendiendo a no compartir propuestas de trabajo a los demás profesores, pero abierta hacia adentro, pues aceptan el trabajo de otros, garantizando con este, que los cuerpos académicos logren su consolidación, situaciones que generan tensión en el ambiente laboral.
\end{abstract}

\section{Palabras clave}

Asociación de profesores, formación de profesores, organización universitaria, academias, formación de investigadores.

\begin{abstract}
The objective of this work is to describe the communicative and social structures found in the groups of researchers that make up the academic bodies in Mexico. A qualitative-phenomenological study was used, where interviews and observations were carried out in a particular context, as well as the analysis of 150 academic products on the subject. The results describe that the academic bodies maintain a closed social and communicative structure, tending not to share work proposals with the other professors, but open inwards, since they accept the work of others, guaranteeing with this, that the academic bodies achieve its consolidation, situations that generate tension in the work environment.
\end{abstract}

\section{Keywords}

Association of professors, teacher training, university organization, academies, training of researchers.

Forma sugerida de citar: Beltrán Poot, Augusto David (2018). La estructura comunicativa y social de los cuerpos académicos en México. Universitas, 28, pp. 79-98. 


\section{Antecedentes}

En México, la figura de cuerpos académicos (CA) surge en 1996, como parte del Programa de Mejoramiento del Profesorado (PROMEP, en la actualidad Programa para el Desarrollo Profesional Docente PRODEP) cuya finalidad es profesionalizar a los Profesores de Tiempo Completo (PTC) para que alcancen las capacidades de investigación-docencia, desarrollo tecnológico e innovación y con responsabilidad social, se articulen y consoliden cuerpos académicos y con ello generen una nueva comunidad académica capaz de transformar su entorno (PRODEP, 2016).

Un cuerpo académico son grupos de profesores de tiempo completo que comparten una o varias Líneas de Generación y Aplicación Innovadora del Conocimiento (LGAC) (investigación o estudio) en temas disciplinares o multidisciplinares y un conjunto de objetivos y metas académicos. Adicionalmente sus integrantes atienden Programas Educativos (PE) en varios niveles para el cumplimiento cabal de las funciones institucionales (PRODEP, 2016).

Las instituciones educativas (IES) que incorporan el trabajo académico por medio de los CA, suponen tener mayores beneficios que les permiten consolidarse como escuelas de calidad, ya que poseen profesores altamente capacitados, que se relacionan entre sí para desarrollar investigaciones, resolver problemáticas del entorno y transmitir sus saberes en las aulas, favoreciendo de esta manera a los estudiantes al propiciar escenarios en los que la teoría y la práctica están fuertemente vinculados.

Sin embargo, estudios como los de Martínez Romo, S. (2005); Pérez, J. (2009); Estrada, I., \& Cisneros, E. (2009); Prieto, M., Valencia, A., Carrillo, J., \& Castellanos, J. (2011) y Magaña Medina, D. E., Surdez Pérez, E. G., Sandoval Caraveo, M. d., \& Aguilar Morales, N. (2015), entre otros, nos permite evidenciar que a lo largo de estos veinte años de implementación del PRODEP, existen experiencias de tensión, incertidumbre, frustración y desgaste emocional y profesional tanto de los profesores que participan en los cuerpos académicos como de los que no. Dichas emociones, son provocadas por las presiones de integración, la carga de trabajo, la búsqueda de recursos para desarrollar investigación o cumplir con los indicadores solicitados, entre otros factores, de aquí el interés por conocer cómo son las estructuras comunicativas y sociales de estos grupos.

En el 2014, la Universidad donde se realiza el estudio contaba con 751 profesores de tiempo completo, de los cuales el 65\% (489) tenía perfil PRO- 
DEP y el 29\% (215) pertenecía al Sistema Nacional de Investigadores. A su vez contaba con 79 cuerpos académicos, de los cuales $21(27 \%)$ se encontraban en formación; 28 (35\%) en consolidación y 30 (38\%) estaban consolidados. Estas unidades académicas favorecían a 97 líneas de generación y aplicación del conocimiento.

Pero, solo 316 (42\%) profesores estaban relacionados directamente con estos grupos, ya que cada CA oscilaba entre 3 y 5 integrantes. 435 (58\%) profesores participan indirectamente o no participan en las metas de dichos conjuntos. Esta situación por una parte, permite distinguir que las decisiones, los acuerdos y las formas de trabajo que se consensuaban en los CA impactaban a los demás profesores.

Debe señalarse, que los cuerpos académicos pueden ser apreciados como mapas textuales de los aspectos políticos, sociales, comunicativos y éticos de la realidad que representan. Estas agrupaciones de investigadores figuran como un sistema que puede referirse así mismo mediante sus comunicaciones y acciones, y es por medio de estas comunicaciones y acciones que se consolida su autodefinición, haciendo de ésta un aspecto normativo dentro de la Institución.

Ante las situaciones que vivían los docentes y percibir que no todos ellos participaban dentro de estas asociaciones, surgieron los siguientes cuestionamientos: ¿Cómo se configuran las estructuras sociales de los CA?, ¿De qué manera logran sus estructuras comunicativas? ¿cuáles estructuras sociales y comunicativas que son normalizadas?

\section{Metodología}

El estudio parte del paradigma cualitativo, bajo el diseño fenomenológico. Considerando como base la teoría de Luhmann, en la que se describe que los sistemas sociales se construyen a partir de la comunicación. De esta manera, se considera posible conocer las estructuras sociales y de comunicación de los CA, porque se encuentran sus comunicaciones. Se considera relevante analizar lo que los mismos investigadores han descrito sobre el tema, así como observar y entrevistar a los profesores universitarios que viven esta experiencia. Los datos son recabados durante el periodo noviembre 2013 a enero 2015.

Se analizaron 150 productos académicos que refieren a las percepciones, significados y formas de trabajo de los CA. Dicha información es obtenida a 
través de bases de datos como Redalyc, ANUIES, COMIE. También se encontraron libros, capítulos de libros y ponencias sobre el tema en diferentes páginas web. La finalidad de este escrutinio consiste en identificar lo que se dice de manera global sobre este tema.

Por otra parte, se estudiaron las estructuras sociales y comunicativas de una IES de una Universidad del sureste de México, que contaba con 41 profesores de tiempo completo (PTC) 16 de ellos integraban los tres cuerpos académicos de la institución, cuyos integrantes oscilaban entre tres y siete participantes. 35 profesores restantes, no configuraban como integrantes de los CA. La finalidad era reconocer de manera concreta lo que en la práctica sucedía, para ello se realizaron observaciones y 28 entrevistas a profesores que participaban en los CA, así como aquellos no configuraban como integrantes.

Para el análisis de datos se organizó la información y se realizaron categorías desde el programa ATLAS.ti, permitiendo de esta manera el cumplimiento de los objetivos planteados.

\section{Resultados}

Todo sistema social o grupo de personas está integrada por una o varias estructuras de aceptabilidad y una configuración comunicativa que permite la interiorización de esta. Por estructura de aceptabilidad, entendemos los aspectos axiológicos que permiten la configuración de un grupo, estos pueden estar enmarcados por cuestiones legales, ideales, creencias, afectivas, entre otra, la finalidad es generar criterios valorativos para la aceptación o rechazo de ideas, métodos o personas que favorezcan o no al grupo.

Por su parte, el lenguaje es el mecanismo por el cual se exterioriza y se regula la autodefinición grupal, inclusive se sanciona a los integrantes del grupo al no adecuarse a las formas de trabajo de los grupos. Los CA pueden definirse como estructuras sociales que contiene en común varias estructuras de aceptabilidad que los definen como grupo académico. Dichas características garantizan en el grupo de académicos su estabilidad y permanencia, estas estructuras de aceptabilidad pueden ser divididas en tres aspectos: políticas, sociales y educativas. 


\section{En cuanto a los aspectos políticos}

Se ha considerado como primera estructura de aceptabilidad la política, debido a que se menciona 560 veces en los artículos de investigación y 80 veces en las entrevistas de los investigadores y docentes universitarios. En la Tabla 1 se describen el número y porcentaje de los términos que se relacionan con este concepto.

Tabla 1

Número y porcentaje de los términos relacionados con el concepto política

\begin{tabular}{|l|l|l|l|l|}
\hline \multirow{2}{*}{\multicolumn{2}{|c|}{ Término }} & \multicolumn{2}{c|}{ Artículos } & \multicolumn{2}{c|}{ Entrevistas } \\
\cline { 2 - 5 } & \multicolumn{1}{|c|}{ No. } & \multicolumn{1}{c|}{$\%$} & No. & \multicolumn{1}{c|}{$\%$} \\
\hline Poder & 252 & 45 & 40 & 50 \\
\hline Norma o regla & 200 & 36 & 21 & 26 \\
\hline Jerarquía & 108 & 19 & 19 & 24 \\
\hline Total & 560 & 100 & 80 & 100 \\
\hline
\end{tabular}

Fuente: Creada a partir de diferentes fuentes

En la tabla anterior se evidencia que tanto la percepción nacional, dictada por los artículos de investigación y la percepción local existe consistencia con el discurso sobre las políticas, ya que en los tres conceptos se mantiene la simetría entre el porcentaje.

El poder es el concepto que más refieren los investigadores o docentes al hablar de la política descrita en el programa PRODEP, los participantes hacen alusión a la imposición por parte de una autoridad. En su mayoría los profesores no evocan a las autoridades de las dependencias a las que pertenecen, más bien a los mandos de un orden mayor, siendo los que se encuentran a cargo de las universidades o en su caso a los responsables del Programa PRODEP, inclusive los que están relacionados con la Secretaría de Educación Pública.

Los investigadores asumen que de acuerdo a la toma de decisiones de estos mandos, es que se da respuesta a las presiones o tendencias internacionales. Sánchez Domínguez (2013, p. 17), describe esta situación al narrar que: 
...los cuerpos académicos como instancias generadoras de conocimiento se establecieron en las IES a partir de la aplicación de ciertas políticas públicas impulsadas por la necesidad de hacer frente a una lógica globalizante de racionalidad pragmática, pero también como una forma de regular el acceso a los recursos públicos.

Esta situación de imposición ha dado como consecuencia que muchos de los cuerpos académicos registrados sean agrupaciones establecidas más por las políticas que por los intereses de generar y/o aplicar una línea de investigación en concreto.

Por otra parte, para los profesores noveles, las políticas declaradas en el programa PRODEP, son normas o reglas que deberán cumplir. Su función como colaboradores de los cuerpos académicos les ha permitido percibir a estos grupos como una plataforma en la que pueden alcanzar una serie de logros profesionales, aunque no tengan un valor para su estabilidad laboral o un beneficio económico. El Mtro. Gerardo describe que un CA es:

...una muy buena idea, una muy buena manera de capitalizar los esfuerzos para que los diferentes académicos podamos trabajar en conjunto. Me siento parte del cuerpo académico, porque, considero que de manera gradual me he ido incorporando y he contribuido en medida de mis posibilidades y de mis circunstancias labores... aunque mi integración en el núcleo base o mi plaza o base sea incierta (E23/02032014).

Otro ejemplo de esta situación lo expresa la Mtra. Martha, ella declara que esta experiencia le ha favorecido, ya que a través del trabajo en CA ha logrado realizar productos académicos que sola o en otras condiciones no pudiera haber logrado. Sin embargo, reconoce que esta situación no es igual para todos. Así lo narra ella:

En realidad mi experiencia profesional es de cinco años, y entonces ciento que al estar en el CA como colaboradora, he podido producir y hacer muchas cosas, en colaboración. Ahora, en este momento de mi carrera eso es perfecto, porque puedo hacer lo que yo quería, cosas que yo no podría hacer si no perteneciera a esta agrupación.

Para mí, está bien, pero veo la posición de los otros profesores, que es de frustración, por no llevar a la práctica lo que ellos quisieran en este momento de su carrera, experiencia y todo, porque aún no son miembros del CA. Creo que para mí en este momento, por mi edad, por el momento que estoy 
viviendo, por buscar hacer mi carrera, la madurez profesional, pues para mí está bien. Tal vez para otros no (E07/21032014).

Se observa y se percibe en el discurso de los docentes o investigadores que las políticas de los CA promueven la creación de jerarquías, que en muchos de los casos generan tensión o malas interpretaciones dentro de la institución. Habrá que reconocer que estos grados no son generados por los Investigadores o profesores de las IES, sin embargo, son estos los que le han resaltado el significado en el ámbito laboral.

Estrada \& Cisneros (2009, p. 10) describen que la jerarquía establecida por el programa PRODEP, es una manera de establecer control entre los docentes. Las autoras afirman que:

...el origen de los CA en la universidad concuerda claramente con una lógica de implementación prospectiva, la cual está asociada a un principio de control jerárquico en cascada ejercido desde la cúspide de quienes implementan la política.

Estas jerarquías por lo menos están definidas por tres situaciones: en cuanto al nombramiento del profesor universitario (investigador, profesor-investigador, profesor de carrera), con respecto a los CA (en formación, en consolidación y consolidado), en cuanto a los logros alcanzados (perfil deseable PRODEP, sin Perfil deseable). Martínez Rodríguez, Villanueva Ibáñez \& Vázquez Mora (2013, p. 35), describen en las conclusiones de su investigación sobre "El trabajo colaborativo de los Cuerpos Académicos", que existe diferencia en la forma de organización entre los CA que se encuentran en formación y los que ya están consolidados. Los investigadores mencionan que:

...durante la interacción de los profesores-investigadores participantes en el grupo focal fue notable un contraste discursivo entre los integrantes de los Cuerpos Académicos en Formación (CAEF) y los Cuerpos Académicos en Consolidación (CAEC) respecto a la forma de trabajo que predomina en los CA. Se observaron diferencias; por una parte respecto al alto nivel de madurez en los acuerdos organizativos para desarrollar el trabajo académico en los CAEC y, por otra, los CAEF expresaron lo complicado que era trabajar de forma colaborativa con los miembros de su cuerpo académico por las diferencias de estilos de trabajo, personalidad e intereses. Estos factores marcaron una inconsistencia interna en las posibilidades de generar trabajo colegiado de forma cooperativa para el cumplimiento de metas y acuerdos mínimos. 


\section{En cuanto a los aspectos sociales}

Un cuerpo académico puede considerarse como un sistema en el cual los integrantes que lo componen tienden por un lado a la realización de sí mismo y por otro, a la contribución de las metas del grupo. Todo esto, por medio de las acciones que han sido aceptados de manera consensuada por los mismos integrantes del CA.

Los cuerpos académicos en muchas ocasiones han sido declarados como sistemas cerrados, esto no quiere decir que sean estáticos o permanentes, tampoco que no permitan la movilidad entre sus miembros. Pues por una parten, existe la posibilidad de cambio, así como la opción de desintegración lo que obligaría a sus participantes cuestionarse sobre la posibilidad de integrarse a otros grupos. De este modo, la movilidad solo podrá realizarse si los integrantes que se van o aquellos que se incorporan comparten en su totalidad los intereses del grupo al que llegan, ya que de no serlo, la estabilidad del CA se verá amenazada o el integrante será rechazado.

Al respecto, Maturana (1996, p. 72) asegura que en un sistema social puede persistir en algunos de sus miembros la presencia de la hipocresía, ya que adoptan o aparentan aceptar acciones que el grupo solicita. Esta situación genera inestabilidad porque la insinceridad siempre se manifiesta en acciones conflictivas debido a la contradicción emocional que implica la hipocresía. El Dr. Germán describe que esta situación puede apreciarse en el clima organizacional, al referirse que por medio del CA se obtiene status, así lo menciona en su discurso:

El CA si influye en el clima organizacional, habría que ampliar un poco el análisis, se vuelve el CA una torre de marfil, los que forman parte del CA obtienen un estatus, y ese estatus a la vez da poder. Un ejemplo al respecto son las asesorías de tesis, que también ya son un criterio para el programa de estímulo docente, o la beca de desempeño, las tesis se reparten entre los integrantes del CA. Entonces si hay una arbitrariedad por parte del CA para favorecer a los que están en los núcleos base. Sabemos que la solvencia del CA es que el núcleo básico esté consolidado, por eso la idea de que las investigaciones sean entre ellos, pero esta disposición o esta necesidad segrega o distancia a otros, cuya participación es menor, que es claramente menor porque no impacta en la imagen del CA, dentro de los estándares de su evaluación (E21/04122014). 
Para comprender un poco la estructura social de los CA, es necesario mencionar que cada asociación está configurada por un grupo de investigadores que se les denomina núcleo base y otro grupo de docentes que son considerados asociados o colaboradores del CA. En cuanto al primer grupo, la institución los considera como personal con estabilidad. De acuerdo a su nombramiento la mayoría son investigadores o profesores-investigadores, tienen doctorado, han obtenido el perfil deseable PRODEP y pertenecen al Sistema Nacional de Investigadores.

El segundo grupo, denominado profesores colaboradores o asociados, son profesores de tiempo completo, no todos tienen la definitividad o plaza laboral, muchos de ellos están por contrato. En cuanto su nombramiento, estos suelen ser en su mayoría profesores de carrera, poseen perfil deseable PRODEP y muy pocos pertenecen al Sistema Nacional de Investigadores.

A través de las entrevistas y las observaciones en la institución se identificó que los CA cuentan con diversas formas de relacionarse. Estas interacciones se han modificado con el pasar del tiempo, pues han respondido a situaciones concretas derivadas de las fuentes evaluadoras de los trabajos de CA, las tendencias institucionales o los intereses de los propios investigadores.

La primera relación que se da en los CA es de orden temporal y normativo, y que fue un poco accidentada, debido a la imposición de formar los CA y por otra la incertidumbre de cómo conciliar las líneas de interés de cada profesor. En este caso, la situación no fue tan dramática, pues los profesores reconocieron lo relevante que era trabajar en equipo; sin embargo, vieron una limitante al distinguir que su interés por ciertos temas que deseaban estudiar, deberían ser olvidados al no estar en la línea de investigación del CA. Así lo describe la Mtra. Lizeth:

Cuando nos pidieron formar cuerpos académicos, yo recuerdo que se dijo en qué área trabajas o das clase, así asignaron a los profesores a los cuerpos académicos. Esto no estaba mal, en el entendido que continuabas con la línea que haz estado trabajando. Lo que no me gusto, es qué a través de los cuerpos académicos mis intereses por otras áreas, porque yo deseaba incorporarme a otras asignaturas, conocer otras áreas, se fueron desvaneciendo, pues ya no podía hacerlos desde el cuerpo académico en el que estoy. Así me quedé (E24/09092014).

Las relaciones creadas a partir del interés de los integrantes del CA, son también de diversas formas. Por una parte, se encuentran las que están en 
función del lugar que ocupan los integrantes del cuerpo académico, otra en relación a la autoridad, experiencia o categoría de quien crea la relación, y una última está en relación a la afinidad entre los miembros.

En cuanto al lugar que ocupan dentro del CA, es evidente en las observaciones y la manera en cómo se organizan para trabajar un proyecto académico. Existen experiencias positivas en las que los CA han generado una relación estrecha, inclusive se han vuelto parte de una familia, la cual busca los medios para construir su hogar, en este caso su espacio de trabajo. Ejemplo de ello se plasma en la narración de Karla (2013, en Cabrera Fuentes \& Díaz Ordaz Castillejos p. 87):

Juntos decidimos construir nuestros propios cubículos con nuestros propios recursos económicos y equiparlos con el apoyo económico del Programa de Mejoramiento del Profesorado. Fuimos blanco de una crítica mordaz, porque nos atrevíamos hacer lo que nunca nadie había hecho: edificar un terreno que era de todos pero a la vez de nadie. Juan Carlos se encargó, como lo hubiera hecho el mejor de los arquitectos, de la obra. La mezcla que une a los tabiques estaba hecha de nuestros aguinaldos y salarios como profesores, cuando el dinero se agotó, empezamos a hacer donaciones y así finalizamos el tan ansiado espacio. Recurrentemente nos preguntamos el motivo que nos impulsa a pasar tanto tiempo en nuestras oficinas, la respuesta es muy simple, porque es también nuestra casa, o nos lo recuerda, ya que hemos llevado muebles y enseres de nuestros hogares: ahí están el comedor y el librero de Lety y Juan, los cuadros de Elsamaría, las plantas de Fernando, las velas de Rosario, los recuerdos de Nancy y mi sofá (Chacón, 2010b, p. 2).

Visto desde la perspectiva de la comunicación, la configuración social tiene como base la comunicación entre los miembros. Escandel Vidal (2014, p. 145) describe que hay comunicación cuando existe intencionalidad; es decir, la comunicación es una actividad voluntaria, por lo que solo se logra la comunicación cuando hay intención de comunicar. En su caso, Luhmann (1998, p. 123) hace una formulación un poco diferente, el teórico menciona que "la comunicación transforma la diferencia entre información y acto de comunicar en la diferencia de la aceptación o el rechazo de la comunicación". Esta diferencia entre rechazo y aceptación está a cargo de lo que el autor considera código.

Escandel Vidal (2014, p. 86) describe que los códigos y la intencionalidad en el proceso de la comunicación, integrada dos componentes: la distan- 
cia personal y la distancia grupal. En cuanto a la primera se refiere a la relación entre dos individuos; esta relación tienen una vertiente subjetiva y otra objetiva. En cuanto a la distancia grupal, es la relación entre dos colectivos; en ésta también se ven manifestadas las percepciones subjetivas y objetivas. Por consiguiente, será necesario describir las escructuras sociales y comunicativas desde las dos perspectivas.

\section{Estructura desde la distancia personal}

Las comunicaciones que se generan entre los integrantes de los CA, se basan en los tipos de relaciones sociales que están presentes, las cuales involucran criterios de status, experiencia laboral o autoridad. Las relaciones sociales de cada miembro se concibe desde la distancia que media entre integrante a integrante. Por ejemplo, considerando que los agentes posean criterios que pueden configurar los códigos comunicativos que dan sentido al $\mathrm{CA}$, los actos comunicativos son menos normativos entre profesores que se encuentren en la misma experiencia laboral o autoridad, mientras que serán más distantes y normativos si existe diferencia en estos criterios.

Escandel Vidal (2014, p. 57) acuña el término distancia social para hacer referencia a la representación que cada participante se forma sobre su relación con el interlocutor, incluyendo en ella los componentes individuales y grupales. Según la autora, la distancia social puede ser analizada por medio de dos dimensiones distintas: la jerarquía y la familiaridad. La primera indica la distancia relativa que resulta de la posición de cada uno de los hablantes de la escala social vigente en un grupo. En el caso de la familiaridad, ésta mide la distancia personal y el grado de conocimiento previo entre los hablantes.

El concepto jerarquía se representa en el eje vertical considerando que corresponde a la posición relativa de un individuo, en este caso un docente, dentro de la escala social de la institución. Estar en el nivel más alto del eje significa que se posee un determinado status social debido al poder, entendido por éste el control de los recursos considerados como valiosos (experiencia laboral, autoridad). Por tanto, pertenecer a un cuerpo académico, no es una característica o atributo que el profesor tiene, sino es una propiedad que se le otorga al ser reconocida por la comunidad o cuerpo académico.

Con relación a la familiaridad, este criterio mide la distancia personal entre dos interlocutores. Poole (1927 citado por Escandel Vidal, 2014, p. 86) 
establece dos indicadores que permiten identificar con claridad la distancia entre estos individuos. El primero es el conocimiento previo, se reconoce que entre mayor tiempo se tenga en la relación menor distanciamiento habrá en los actos comunicativos. Mientras que habrá más distanciamiento si existe desconocimiento de alguno de los interlocutores.

El segundo indicador es la empatía; es decir, se reconoce menor distanciamiento si los individios comparten ideas, formas de trabajo e intereses, mientras que habrá mayor distanciamiento si no existierá motivos por los cuáles estén integrados.

Es importante considerar que la relación entre ambos indicadores en la mayoría de los casos es simétrica, lo que representa que existe mayor familiaridad entre dos individuos si lleva ya un tiempo determinado de conocerse y si comparten intereses personales o laborales. En la Figura 1 se representa el modo cómo se relacionan los integrantes del CA, considerando criterios de jerarquía y familiaridad. Los círculos pequeños representan a los integrantes del núcleo base, y los triángulos a los profesores que son asociados.

\section{Figura 1}

\section{Relación social entre los integrantes del CA}

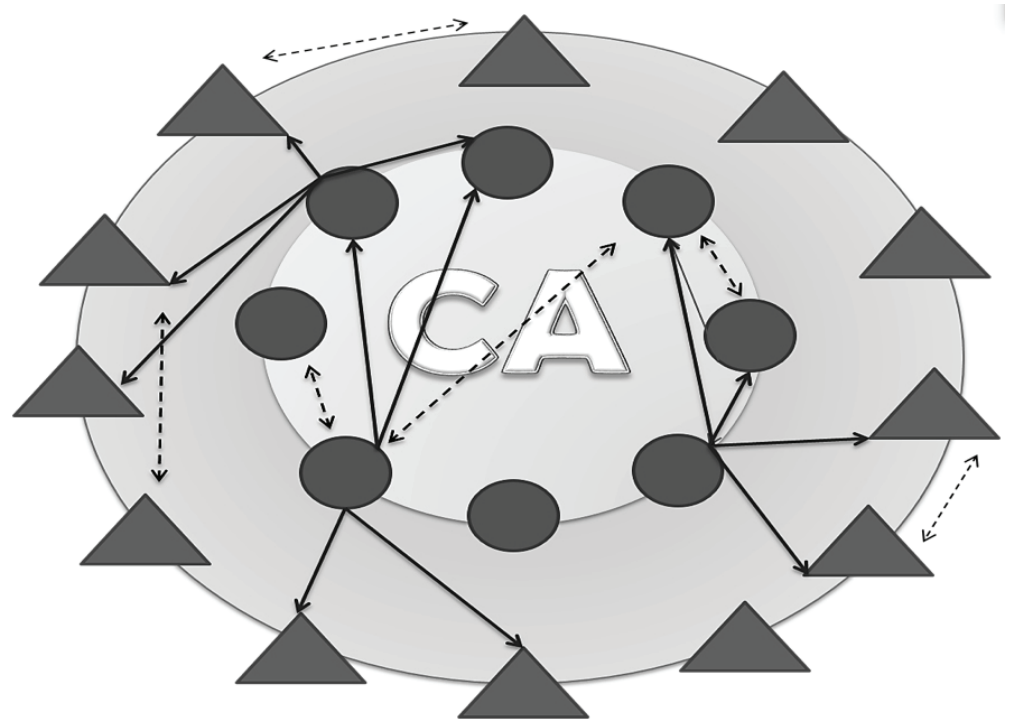

Fuente: Elaboración propia 
En la Figura anterior se puede apreciar que existen dos tipos de relaciones sociales entre los integrantes del CA, una es de forma unilateral y otra bilateral. Llama la atención que en ambos casos la relación es iniciada por un investigador o profesor del núcleo base. En las relaciones unilaterales se puede apreciar que existe una asimetría entre el aspecto jerárquico y la familiaridad; es decir, quien propicia o dirige el nivel o control de las relaciones sociales es el que posee la jerarquía máxima, pero existe poca o nula familiaridad con los demás interlocutores.

Las características comunes de este tipo de relaciones son: quien genera, orienta y termina el acto comunicativo es el agente que posee la mayor jerarquía, en este caso depende de la experiencia laboral o cargo que tenga el profesor.

En la mayoría de los casos los procesos comunicativos son unidireccionales y no se acepta retroalimentación, los individuos que poseen menor jerarquía asumen los comunicados y actúan bajo lo acordado. Ejemplo de esto, lo narra el Mtro. Juan:

La dinámica con ellos (refiriéndose algunos de los investigadores del núcleo base), es que tienen una idea o ya empezaron un proyecto, te dicen lo que necesitan y te dan los recursos para hacerlo. Hago lo que me piden, ya sea el artículo o la ponencia, base de datos, les comento que les parece, ellos dicen perfecto va, sino te lo regresan y corriges.

En otras ocasiones, es complicado porque no tenemos el mismo lenguaje, cuando trabajo con un miembro del núcleo base, habla mucho y me pierdo, habla mucho y no me dice cosas muy concretas, te dice que es lo que quiere, tal vez sea bueno, pero no me ajusto a ese tipo de dinámica, me gusta más cuando llegamos con los asociados y podemos trabajar desde el principio la idea del proyecto (E16/1310102014).

Esta situación es lógica, si partimos de la idea de que el status, la experiencia laboral y la autoridad, denotan un criterio de expertos de quien los porta; sin embargo, en este contexto se empiezan a identificar los códigos comunicativos presentes en la institución, dejando de manifiesto que es importante establecer y diferenciar los aspectos jerárquicos.

Con respecto a la segunda forma de socializar y comunicarse, pueden existir actos comunicativos que tienen una simetría jerárquica, familiaridad 
o con ambas. Este es el caso de los acuerdos entre los integrantes del núcleo base o los acuerdos entre los asociados.

No obstante, puede haber situaciones en las que las relaciones de tipo bilateral, se presenten entre docentes con un distanciamiento jerárquico y con una alta familiaridad o viceversa.

Las características comunes de estos grupos son; por un lado, ambos interlocutores están en disposición de desarrollar actos comunicativos, por lo que se genera un ambiente de intercambio de intereses, expectativas y proyectos laborales, las funciones y las formas de trabajo se realizan mediante acuerdos más equitativos que los anteriores, ya que se comparten responsabilidades por igual. Por ejemplo, el Mtro. Gerardo, describe que existe un interés, de los profesores o investigadores del núcleo base, por apoyar a los docentes noveles, esta relación se basa más en el aspecto de la empatía, y no en el ámbito de la jerarquía, debido a que se han formado vínculos gracias al trabajo como asesor de tesis o haber participado en algún proyecto académico. Esto es lo que menciona al respecto:

Otra cosa interesante, ese velar por los asociados a veces lo veo, lo entiendo, más en términos personales, de amistad, no tanto como de academia, si me preocupo por ti como asociado es porque a lo mejor, no es mi amigo pero te aprecio, porque hay una relación, como asesor o por lo que fuera, en ese sentido más que en términos académicos, y no como integrante del núcleo base comparto con mis colegas del núcleo base un plan de desarrollo para que los asociados aprendan apuntalar el CA (E08/02042014).

\section{Estructura desde la distancia grupal}

La situación anterior coincide con la relación entre los diferentes cuerpos académicos (ver Figura 2). Los aspectos de jerarquía y la familiaridad se encuentran presenten debido a la asignación de las categorías de los CA (en formación, en consolidación y consolidados). Además del discurso sobre el monto de financiamiento de las investigaciones, la cantidad y tipos de productos realizados por CA. Dando como consecuencia una lucha entre los CA por conservar el status de cuerpo académico, lo que a la larga representaría una posición de poder. 
Figura 2. Relación entre integrantes de diferentes CA

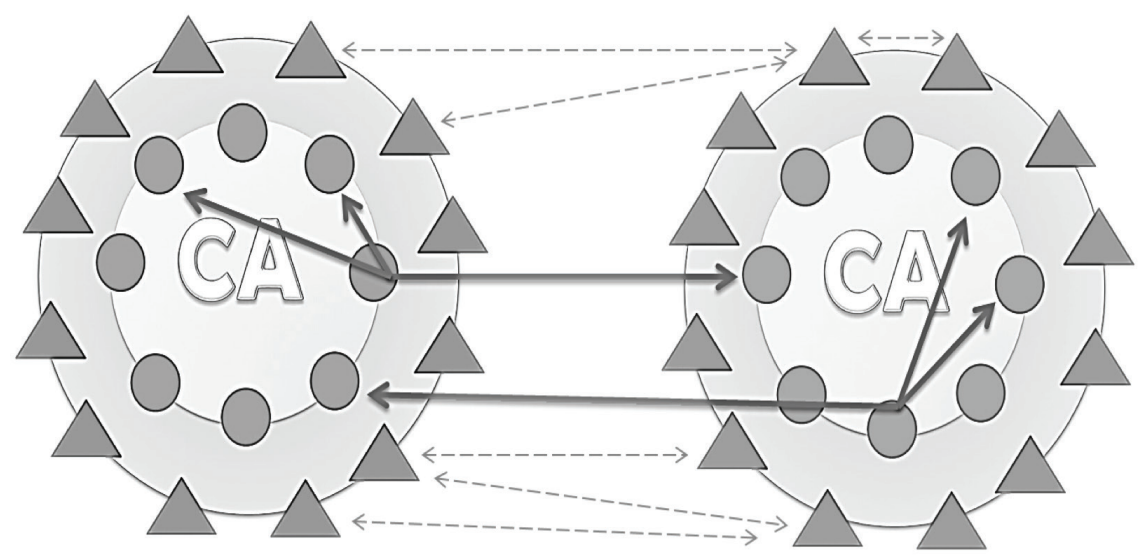

Fuente: Elaboración propia

No obstante, las observaciones y las entrevistas no han permitido identificar cómo son las relaciones entre los integrantes del CA y los miembros de otros cuerpos académicos de otras dependencias. Sin embargo, hay datos que permiten inferir que la jerarquía es un elemento indispensable para el desarrollo de estas relaciones, debido a que las políticas educativas o las instancias evaluadoras tienen como indicador válido solo las relaciones o redes establecidas con CA del mismo nivel.

\section{En cuanto a los aspectos académicos}

La Universidad históricamente ha sido organizada con la finalidad de transmitir y buscar en el estudiante el aprendizaje del conocimiento y el desarrollo de habilidades, en la actualidad las tendencias han hecho de estos espacios educativos, más que sitios pasivos de aprendizaje, sean lugares que activen tanto al docente como al estudiante con la finalidad de dar respuesta a problemas que se sitúan en su contexto.

Para Zúñiga, Barona, Ponce, Torres \& Zorrilla (2011, p. 130), el rol del docente va asociado a la función social que en cada momento histórico le asigna el sistema educativo; por lo que es necesario considerar que la identidad del docente se va conformando a partir de una estructura que le otorga 
su sentido y direccionalidad. Ante esto, es importante recalcar que antes del programa PRODEP la acción de transmisión de conocimiento permitía que el docente pudiera realizar esta función solo; pero el contexto actual requiere que el profesor una voluntades con otros docentes no solo para transmitir conocimientos, sino para generar y llegar a acuerdos que respondan a los cuestionamientos de qué, cómo y para qué enseñar.

Los cuerpos académicos han permitido identificar a los docentes como sujetos sociales que construyen sus caminos a través de las experiencias de trabajo, los procesos evaluativos a los que se enfrentan y los intercambios de formas de trabajo con otros cuerpos académicos. Esto a través de compartir significados por medio de marcos comunes, donde los procesos intercomunicativos determinan los estilos, sentidos y significados de la práctica (Zúñiga, Barona, Ponce, Torres \& Zorrilla, 2011, p. 130). De igual forma, los autores para reafirmar su percepción sobre el trabajo en los CA, citan a Cedrato (2009, p. 38) y afirman que:

...por consiguiente, el trabajo colaborativo al interior de los cuerpos académicos contribuye al desentrañamiento intelectual, puesto que implica disposición al debate y a la apertura del pensamiento de otros para escuchar algo diferente, así como contar con disposición para aceptar diversas posturas de un mismo tema, de la creatividad para producir.

Involucra aceptar que todos tienen una forma particular de pensar, de esta forma una diversidad de pensamiento enriquece y problematiza una situación, que las ideas son relativas y versátiles, que se construyen en un proceso de retroalimentación. La tolerancia y el respeto del pensamiento del otro aunque difiera del propio, es un ejercicio muy valioso para lograr la creación intelectual colectiva.

Las relaciones con base a los aspectos académicos tienen una doble finalidad; por una parte se encuentra el desarrollo de una línea de investigación que en muchas ocasiones responde a un aspecto más personal; y por otra, a la disposición de generar un conocimiento que permita al docente nutrir a su estudiante de recursos para desenvolverse en un contexto determinado, o colaborar con el desarrollo de un cuerpo colegiado, siendo éste un aspecto más social.

Por otra parte, en los CA se declaraban líneas de investigación que integraban las áreas de interés de los profesores que eran miembros del grupo. Esta situación, al principio solo fue de nombre, pues los profesores realiza- 
ban sus investigación de manera independiente, si en su caso eran investigadores, porque de no serlo, continuaban con la impartición de clases. Ejemplo de esto lo menciona el Mtro. Pablo:

...lo que pasa es que, cada quien a avanzado en su propia línea de investigación, esto es reflejo de un trabajo que se ha hecho en toda la vida académica; por ejemplo, yo he estudiado casi toda mi vida éste tema, dedicarme a una línea de investigación que no ha sido la mía, me genera conflicto, pues no es lo que yo deseo realmente.

Esto es lo que sucede al interior del CA, cada quien tiene sus propios intereses, hablando en temas de investigación, y es muy dificil poder hacer que el otro deje su área por atender la mía, o viceversa (E18/12092014).

Con el pasar del tiempo los criterios de trabajar en cuerpo académico obligó a generar mecanismos que no eran sanos; ya que se realizaban permutas de nombre para aparentar el trabajo colegiado. De esta manera, se garantizaba que los investigadores o docentes siguieran trabajando en su área de interés y lograran sumar puntos para sí y el CA, pero no desarrollar productos en conjunto. La Mtra. Lizeth menciona al respecto:

Cada profesor tiene su línea de trabajo, nos han hecho observaciones de que se nota las líneas de investigación de cada uno, inicialmente cuando empezó el trabajo de los CA, así era, no había los criterios de trabajar más colegiadamente, es decir, que debe haber dos profesores del mismo CA en cada trabajo y cuando nos hacen esta obsevación, por un tiempo seguimos con la misma dinámica, pero hacemos un poco de ficción, si voy a publicar un artículo de mi tema, pongo a otro compañero, aunque no este enterado de lo que yo estoy haciendo, y esto se da de la misma manera con el otro. Esta situación va aparentar que estamos trabajando colegiadamente. Pero esto es una ficción. ¡Ahora! esto es un criterio que está establecido por quien sabe quién, y tendrá que demostrar que es valido; pues cree que al obligarnos a trabajar en dos de la misma línea sera mejor (E15/06072014).

A pesar de estas dificultades, los beneficios del trabajo en cuerpos académicos se ve apreciado en diferentes aspectos: mayor formación o habilitación docente, los programas educativos son calificados con criterios de calidad por las fuentes evaluadoras, los estudiantes se ven beneficiados con escenarios de aprendizajes más reales y menos teóricos, se les involucra en proyectos de investigación que de una manera u otra acorta la distancia so- 
cial entre el investigador-docente y estudiante, situaciones que sugieren la preocupación por un ejercicio docente responsable que pretende dar respuesta a las necesidades de políticas globales.

\section{Conclusiones}

Los datos recabados describen que las estructuras sociales están fuertemente ancladas a los aspectos políticos, sociales y académicos que representan la nueva labor docente del profesor universitario mexicano.

Las claves comunicativas de los cuerpos académicos, están identificados por aspectos de jerarquía y familiaridad. Siendo en este caso la jerarquía el más influyente, debido que para las universidades mexicanas, así como para el programa PRODEP las diferencias son importantes, pues se promueven a través de los grados académicos, productos realizados o nivel de cuerpos académicos al que se pertenece.

No obstante, los resultados de las entrevistas, así como del análisis de documentos ofrecen datos para considerar que la alienación de los profesores universitarios se agudiza cuando estos se orientan al proceso de producción y de evaluación con la finalidad de alcanzar beneficios económicos, de poder y de estatus. Ocasionando la pérdida de interés por el auténtico desarrollo de una línea de generación y/o aplicación del conocimiento, así como la actitud de mejora que ofrece la evaluación.

Los datos describe que cada CA y cada DES a lo largo de este tiempo de implementación del programa, han desarrollado mecanismos para asegurar el estatus hasta ahora alcanzado. Generando que el profesor universitario modifique su labor docente, ya que al compartir esta función con la investigación vive tensionado por la preocupación de desarrollar diferentes productos académicos que a su vez son indicadores de la eficacia y eficiencia de su quehacer, en la evaluación.

El trabajo elaborado reconoce los beneficios que se obtienen al trabajar bajo la modalidad de cuerpos académicos; sin embargo, comprendemos que existen áreas de mejora que no dependen de la normativa o los ideales del Programa, más bien, son responsabilidad de los integrantes, sobre todo coordinadores o responsables de los CA el promover mecanismos más transparentes y equitativos para todos los docentes que desean participar en estos grupos. 
Por último, la comunicación es un recurso que ha permitido la normalización tanto del trabajo de los cuerpos académicos, como del Perfil deseable. Por tanto, se debe hacer un doble esfuerzo por las autoridades universitarias y los responsables de los CA para integrar, permitir y apoyar para que los demás docentes que no se encuentren en estos grupos participen en esta dinámica, garantizando una sinergia de trabajo más justa.

\section{Bibliografía}

Cabrera, J., \& Díaz, E. (2013). Construyendo una realidad colectiva. En J. Cabrera Fuentes, \& L. Pons Bonals, Configuraciones narrativas de grupos y cuerpos académicos en el campo de la investigación educativa (pp. 6788). Barcelona: Octaedro.

Escandel, M. (2014). La comunicación: lengua, cognición y sociedad. Madrid: Akal. Estrada, I., \& Cisneros, E. (2009). Origen, reestructuración y desarrollo de los Cuerpos Académicos en una universidad pública del sureste de México. Mérida, Yucatán, México.

Luhmann, N. (1998). Teoría de la sociedad y peagogía. Barcelona: Paidos.

Magaña Medina, D. E., Surdez Pérez, E. G., Sandoval Caraveo, M. d., \& Aguilar Morales, N. (2015). Sindrome de Desgaste Emocional y factores asociados en profesores de Cuerpos Acdémicos. Tabasco: UJAT.

Martínez Rodríguez, R., Villanueva Ibáñez, M., \& Vázquez Mora, J. A. (2013). Factores de Favorecen el trabajo colaborativo en los cuerpos académicos desde la opinión de un grupo de profesores-investigadores de la educación superior tecnológica. En F. Santillán Campos, Los cuerpos académicos en Iberoamérica. Gestión y desarrollo (pp. 28-36). Jalisco: Umbral.

Martínez Romo, S. (2005). Grupos, tribus, cuerpos y redes académicas; vicisitudes y retos de las políticas para la educación superior. En M. Fresan, Repensando la Univesidad. 30 años de trabajo académico de innovación en México (pp. 321-329). México: UAMX.

Maturana, H. (1996). La realidad ¿objetiva o construida? México: Antrhotpos.

Pérez, J. (septiembre-diciembre de 2009). El efecto frankenstein: las políticas educativas mexicanas y su impacto en la profesión académica. Espiral. Estudios sobre Estado y Sociedad, XVI(46), 61-95.

Prieto, M., Valencia, A., Carrillo, J., \& Castellanos, J. (2011). Docentes: entre el malestar y la incertidumbre. En R. Pérez, \& I. Monfredini, Profesión 
académica: mecanismos de regulación, formas de organización y nuevas condiciones de producción intelectual (pp. 221- 234). Guadalajara, Jalisco, México: Universidad Guadalajara.

Programa para el Desarrollo Profesional Docente, para el Tipo Superior (PRODEP) (2016), Manual de operaciones, México. SEP. Recuperado de https:// goo.gl/yoPRU2 (23-05-2016).

Sánchez Domínguez, J. P. (2013). Entre políticas y realidades: un análisis sobre los Cuerpos Académicos. En F. Santillán Campos, Los cuerpos académicos en Iberoamérica. Gestión y desarrollo (pp. 15-21). Jalisco, México: Umbral.

Zúñiga, O., Barona, C., Ponce, O., Torres, S., \& Zorrilla, M. (2011). La enseñanza experta en contexto universitario. ¿qué se deja fuera en la regulación del trabajo académico? En R. Pérez, \& I. Monfredi, Profesión académica: mecanismos de regulación, formas de organización y nuevas condiciones de producción intelectual (pp. 107-132). Guadalajara, Jalisco, México: Universidad de Guadalajara.

Fecha de recepción: 2017/II/24; fecha de aceptación: 2018/02/16;

fecha de publicación: 2018/03/01 